\title{
Protocol for the EARCO Registry: a pan-European observational study in patients with $\alpha_{1}$-antitrypsin deficiency
}

\begin{abstract}
Timm Greulich (10) ${ }^{1}$, Alan Altraja $\mathbb{1}^{2,3}$, Miriam Barrecheguren ${ }^{4}$, Robert Bals $\mathbb{1}^{5}$, Jan Chlumsky ${ }^{6}$, Joanna Chorostowska-Wynimko $\mathbb{1}^{7}$, Christian Clarenbach $\mathbb{1}^{8}$, Luciano Corda9, Angelo Guido Corsico ${ }^{10}$, Ilaria Ferrarotti (i) ${ }^{10}$, Cristina Esquinas ${ }^{4}$, Caroline Gouder ${ }^{11}$, Ana Hećimović ${ }^{12}$, Aleksandra Ilic ${ }^{13,14}$, Yavor Ivanov ${ }^{15}$, Sabina Janciauskiene ${ }^{16}$, Wim Janssens (17, ${ }^{17}$, Malcolm Kohler ${ }^{8}$, Alvils Krams ${ }^{19,20}$, Beatriz Lara (1) ${ }^{21}$, Ravi Mahadeva ${ }^{22}$, Gerry McElvaney ${ }^{23}$, Jean-François Mornex ${ }^{24,25}$, Karen O'Hara ${ }^{26,27}$, David Parr ${ }^{21}$, Eava Piitulainen ${ }^{28}$, Karin Schmid-Scherzer ${ }^{29}$, 'Niels Seersholm ${ }^{30}$, Robert A. Stockley ${ }^{31}$, Jan Stolk ${ }^{32}$, Maria Sucena ${ }^{33}$, Hanan Tanash ${ }^{28}$, Alice Turner (103,35, Ruxandra Ulmeanu ${ }^{36,37}$, Marion Wilkens ${ }^{38}$, Arzu Yorgancioğlu ${ }^{39}$, Ana Zaharie ${ }^{40}$ and Marc Miravitlles $\mathbb{1}^{4}$, on behalf of the EARCO Clinical Research Collaboration
\end{abstract}

\section{ABSTRACT}

Rationale and objectives: Alpha-1 antitrypsin deficiency (AATD) is a genetic condition that leads to an increased risk of emphysema and liver disease. Despite extensive investigation, there remain unanswered questions concerning the natural history, pathophysiology, genetics and the prognosis of the lung disease in association with AATD. The European Alpha-1 Clinical Research Collaboration (EARCO) is designed to bring together researchers from European countries and to create a standardised database for the follow-up of patients with AATD.

Study design and population: The EARCO Registry is a non-interventional, multicentre, pan-European, longitudinal observational cohort study enrolling patients with AATD. Data will be collected prospectively without interference/modification of patient's management by the study team. The major inclusion criterion is diagnosed severe AATD, defined by an AAT serum level $<11 \mu \mathrm{M}\left(50 \mathrm{mg} \cdot \mathrm{dL}^{-1}\right)$ and/or a proteinase inhibitor genotype ZZ, SZ or compound heterozygotes or homozygotes of other rare deficient variants. Assessments at baseline and during the yearly follow-up visits include lung function testing (spirometry, body plethysmography and diffusing capacity of the lung), exercise capacity, blood tests and questionnaires (symptoms, quality of life and physical activity). To ensure correct data collection, there will be designated investigator staff to document the data in the case report form. All data will be reviewed by the EARCO database manager.

Summary: The EARCO Registry aims to understand the natural history and prognosis of AATD better with the goal to create and validate prognostic tools to support medical decision-making.

@ERSpublications

The EARCO Registry is a non-interventional, multicentre, pan-European, longitudinal observational cohort study enrolling patients with AATD to elucidate the natural history, pathophysiology, genetics and prognosis of this condition http://bit.ly/369ScCc

Cite this article as: Greulich T, Altraja A, Barrecheguren M, et al. Protocol for the EARCO Registry: a pan-European observational study in patients with $\alpha_{1}$-antitrypsin deficiency. ERJ Open Res 2020; 6: 00181-2019 [https://doi.org/10.1183/23120541.00181-2019]. 


\section{Introduction}

Alpha-1 antitrypsin deficiency (AATD) is a common, but under-recognised genetic condition that affects approximately 1 in 2000 to 1 in 5000 individuals in European countries, and predisposes to early-onset emphysema and liver disease $[1,2]$. AAT is mainly produced in the liver, and its function is to protect tissues, especially the lung, against proteolytic damage from serine proteinases including neutrophil elastase [3]. To date, $>100$ variant alleles of the AAT gene have been described, but the Z allele (p.E366 K, c.1096G $>A, r$ rs 28929474) is the most prevalent severe deficiency gene leading to lung and liver disease. The $\mathrm{Z}$ protein forms polymers that remain trapped within the rough endoplasmic reticulum of hepatocytes leading to reduced levels of AAT in the bloodstream [4]. The liver disease arises secondary to accumulation of the protein in hepatocytes, whereas the imbalance between low blood and hence tissue levels of AAT and neutrophil elastase in the lung increases the risk of emphysema [1]. Although tremendous improvements have been made in the understanding of the pathophysiology of the disease in recent years, many questions remain unanswered.

The natural history of AATD patients is not well known, and there is a lack of prognostic tools to support medical decision-making, such as early referral for lung transplantation [5]. Although the vast majority of patients with severe deficiency share the same genetic disorder ( ${ }^{\star}{ }^{\star} \mathrm{ZZ}$ genotype; PI standing for proteinase inhibitor), the prevalence and severity of liver and respiratory disease vary markedly. Genetic and environmental cofactors have been suspected, but few have yet been identified [6, 7].

Augmentation therapy, when assessed using computed tomography (CT) lung densitometry, has been shown to slow the progression of emphysema significantly [8-10]. However, its impact on forced expiratory volume in $1 \mathrm{~s}\left(\mathrm{FEV}_{1}\right)$ decline, quality of life and mortality has not been definitively established. Consequently, the efficacy of augmentation therapy is still debated [11], and the cost of therapy is not reimbursed in all European countries $[12,13]$. Furthermore, most studies on AATD have focused on the $\mathrm{ZZ}$ population, and the impact of other rarer variants on outcomes is largely unknown [14].

The answer to these questions requires the access to large cohorts of patients and cannot be addressed solely at individual country level. This was the main reason to initiate a European Alpha-1 Clinical

Affiliations: 'University Medical Centre Giessen and Marburg, Philipps-University, Dept of Medicine, Pulmonary and Critical Care Medicine, Member of the German Centre for Lung Research (DZL), Marburg, Germany. ${ }^{2}$ Pneumology Dept, Tartu University, Tartu, Estonia. ${ }^{3}$ Lung Clinic, Tartu University Hospital, Tartu, Estonia. ${ }^{4}$ Pneumology Dept, Hospital Universitari Vall d'Hebron/Vall d'Hebron Research Institute (VHIR), CIBER de Enfermedades Respiratorias (CIBERES), Barcelona, Spain. ${ }^{5}$ Dept of Internal Medicine V Pulmonology, Allergology, Intensive Care Medicine, Saarland University Hospital, Homburg, Germany. ${ }^{6}$ Dept of Pneumology, Thomayer Hospital, First Faculty of Medicine, Charles University, Prague, Czech Republic. ${ }^{7}$ Dept of Genetics and Clinical Immunology, National Institute of Tuberculosis and Lung Diseases, Warsaw, Poland. ${ }^{8}$ Division of Pulmonology, University Hospital Zurich, Zurich, Switzerland. ${ }^{9}$ Dept of Internal Medicine, Respiratory Disease Unit, Spedali Civili, Brescia, Italy. ${ }^{10}$ Dept of Internal Medicine and Therapeutics, Pneumology Unit IRCCS San Matteo Hospital Foundation, University of Pavia, Pavia, Italy. "Dept of Respiratory Medicine, Mater Dei Hospital, Msida, Malta. ${ }^{12}$ Dept of Respiratory Diseases "Jordanovac", University of Zagreb, School of Medicine, Zagreb, Croatia. ${ }^{13}$ Clinic for Pulmology, Clinical Center of Serbia, Belgrade, Serbia. ${ }^{14}$ Faculty of Medicine, University of Belgrade, Belgrade, Serbia. ${ }^{15}$ Pulmonary Clinic, University Hospital, Pleven, Bulgaria. ${ }^{16} \mathrm{Clinic}$ for Pneumology, DZL, Medical University Hannover, Hannover, Germany. ${ }^{17}$ Katholieke Universiteit Leuven, Laboratory of Respiratory Diseases, Dept of Chronic Disease, Metabolism and Ageing, Leuven, Belgium. ${ }^{18}$ University Hospitals Leuven, Department of Respiratory Diseases, Leuven, Belgium. ${ }^{19}$ Faculty of Medicine, University of Latvia, Riga, Latvia. ${ }^{20}$ Riga East University Hospital, Riga, Latvia. ${ }^{21}$ Dept of Respiratory Medicine, University Hospitals of Coventry and Warwickshire, Coventry, UK. ${ }^{22}$ Respiratory Medicine, Cambridge University Hospitals NHS Foundation Trust, Cambridge, UK. ${ }^{23}$ Irish Centre for Rare Lung Diseases, Royal College of Surgeons in Ireland, Beaumont Hospital, Dublin, Ireland. ${ }^{24}$ Hospices Civils de Lyon, Service de Pneumologie, Hôpital Louis Pradel, Lyon, France. ${ }^{25}$ Université de Lyon, Université Lyon 1, UMR754, INRA, Lyon, France. ${ }^{26}$ Alpha-1 UK Support Group, Droitwich, UK. ${ }^{27}$ Alpha-1 Global, Miami, FL, USA. ${ }^{28}$ Dept of Respiratory Medicine and Allergology, Skåne University Hospital, Lund University, Malmö, Sweden. ${ }^{29}$ Dept of Internal Medicine II and Pulmonology, Wilhelminenspital, Medical University of Vienna, Vienna, Austria. ${ }^{30}$ Dept of Internal Medicine, Herlev and Gentofte University Hospital, Hellerup, Denmark. ${ }^{31}$ Lung Investigation Unit Medicine, University Hospitals Birmingham NHS Foundation Trust Queen Elizabeth Hospital Birmingham, Birmingham, UK. ${ }^{32}$ Dept of Pulmonology, Leiden University Medical Center, Leiden, The Netherlands. ${ }^{33}$ Pulmonology Dept, Centro Hospitalar de São João, Porto, Portugal. ${ }^{34}$ Respiratory Medicine, University Hospitals Birmingham NHS Foundation Trust, Birmingham, UK. ${ }^{35}$ Institute of Applied Health Research, University of Birmingham, Birmingham, UK. ${ }^{36}$ Marius Nasta Institute, Bucharest, Romania. ${ }^{37}$ Faculty of Medicine, University of Medicine Oradea, Bucharest, Romania. ${ }^{38}$ Patientenorganisation Alpha1 Deutschland e.V., Gernsheim, Germany. ${ }^{39}$ Dept of Pulmonary Diseases, Celal Bayar University, Faculty of Medicine land the GARD Executive Committeel, Manisa, Turkey. ${ }^{40}$ Dept of Pneumophthisiology, University of Medicine and Pharmacy, "Marius Nasta" Institute of Pneumophthisiology, Bucharest, Romania.

Correspondence: Timm Greulich, Dept of Medicine, Pulmonary and Critical Care Medicine, PhilippsUniversity Marburg, 35043 Marburg, Germany. E-mail: greulichdastaff.uni-marburg.de 
Research Collaboration (EARCO) [15]. EARCO is an initiative under the umbrella of the European Respiratory Society (ERS) and its clinical research collaborations, pan-European, multicentre research networks of different fields of respiratory diseases [16]. The initiative brings together multiple stakeholders including researchers, healthcare providers, patients and industry with the aim of advancing understanding through clinical and scientific research and improving the quality of life of patients with the deficiency.

EARCO takes advantage of existing AATD registries that have been developed at the national and international level. Several countries have established registries in which AATD patients are included and followed-up with clinical and biological data collected [14, 17-23]. However, these registries differ in terms of inclusion criteria, data collected and frequency and extent of follow-up. Within EARCO, we will harmonise the data collection process and assess the quality of the data entered into the database. This article describes the EARCO registry protocol: a pan-European multicentre observational study in patients with alpha-1 antitrypsin deficiency.

\section{Study objectives}

The study objectives are

- to develop a pan-European, multicentre AATD registry incorporating baseline data from $\geqslant 3000$ individuals during the first 3 years of the registry;

- to harmonise the data collection process between existing national registries and to ascertain high quality of the data by monitoring entered data closely;

- to generate longitudinal long-term, high-quality clinical data covering a pan-European population of AATD individuals of all age groups and all stages of disease severity;

- to understand the natural history and prognosis of AATD better with the goal to create and validate prognostic tools to support medical decision-making;

- to investigate the effect of AAT augmentation and other therapies on the progression of emphysema and to examine their potential impact on clinical and functional outcomes, such as $\mathrm{FEV}_{1}$, quality of life and mortality in a "real-life" population;

- to learn more about the course of the disease in patients suffering from severe AATD with genotypes other than $\mathrm{PI}^{\star} \mathrm{ZZ}$.

\section{Study design}

The EARCO Registry is a non-interventional, multicentre, pan-European, prospective, observational cohort study enrolling patients with AATD, as confirmed by biochemical and genetic data. Study centres are distributed in $>20$ European countries (figure 1) and include centres in primary, secondary and tertiary care, according to national arrangements. Current countries have been selected by personal contacts and invitations of all steering committee members. We will step up efforts in recruiting more countries through presentations on congresses, meetings and through the webpage (www.earco.eu). The registry is open to every clinician who manages AATD patients. Patients will be managed according to their local procedures and policies and without any clinical intervention from the study team. All patients will provide written informed consent to participate, and participating investigators will collect data prospectively. The following domains are covered: demographics, proteinase inhibitor genotype and other laboratory analyses, comorbidities, lung function, respiratory symptoms, ultrasound-based elastography of the liver, exacerbations of respiratory disease, quality of life, physical activity, chest CT (as applicable) and treatment.

The study protocol has received central ethics approval by the research ethics committee of the Vall d'Hebron University Hospital of Barcelona, Spain (PR(AG)480/2018), and the study database will be hosted in the Vall d'Hebron Research Institute (Barcelona). The study website is located at www.EARCO. eu. The registry was developed according to recommendations on the design, implementation, governance and long-term sustainability of disease registries in the European Union (EU) [24, 25]. We expect to collect detailed information from $\sim 1000$ patients from $\geqslant 10$ countries during the first year after creation of the registry, expanding to 3000 from $>20$ countries over the following 2 years.

\section{Population}

The study population will consist of individuals with diagnosed severe AATD regardless of the clinical features and severity. The inclusion criteria are a diagnosis of AATD, defined as AAT serum level $<11 \mu \mathrm{M}$ $\left(50 \mathrm{mg} \cdot \mathrm{dL}^{-1}\right)$ and/or proteinase inhibitor genotypes $\mathrm{ZZ}, \mathrm{SZ}$ and compound heterozygotes or homozygotes of other rare deficient variants. The only exclusion criterion is lack of patient consent. Patients will be discontinued from the study if the informed consent is withdrawn by the patient or his/her legal custodian. If a patient withdraws from the study, the investigator will undertake every possible effort to determine the primary reason for a patient's withdrawal and record this information on the case report form (CRF). 


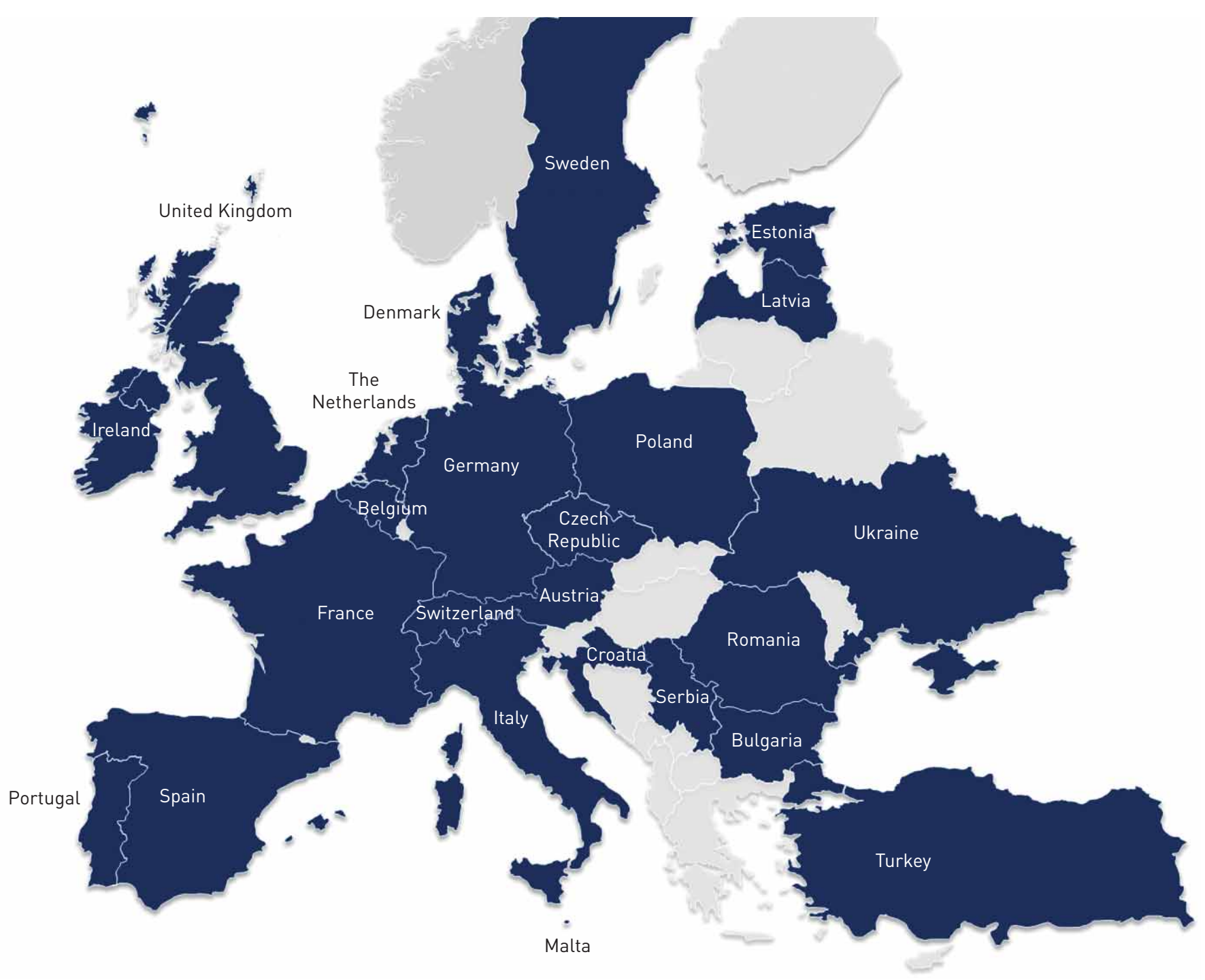

FIGURE 1 European countries in which study centres are located.

For patients who are lost to follow-up (i.e. those patients whose status is unclear because they fail to appear for study visits without stating an intention to withdraw), the investigator should show "due diligence" (if allowed by national ethics committee standards) by documenting in the source documents steps taken to contact the patient, e.g. dates of telephone calls, registered letters, etc.

\section{Visit schedule and assessments}

Written informed consent form will be obtained at the baseline visit. All available information will be collected according to the CRF. Follow-up visits will be scheduled on a yearly basis ( \pm 3 months) and assessments, as described in the following sections, will be performed and recorded in the CRF ((b) represents baseline only) (figure 2). Because the registry reflects standard assessments in many different countries and healthy individuals (fulfilling the inclusion criteria) will be included, only the participant's sociodemographics and basic information about the presence/type of lung or liver disease will be absolutely mandatory. All other assessments are not mandatory, but the data manager will request a minimal set of clinical data through queries to the investigators.

\section{Patient sociodemographics}

The following data will be collected from the patient's medical history: date of birth (b), sex (b), race and ethnicity (b), height, weight, smoking history, alcohol consumption, working status (including relevant occupational exposures and type of agent), year of diagnosis of AATD (b), information about family 


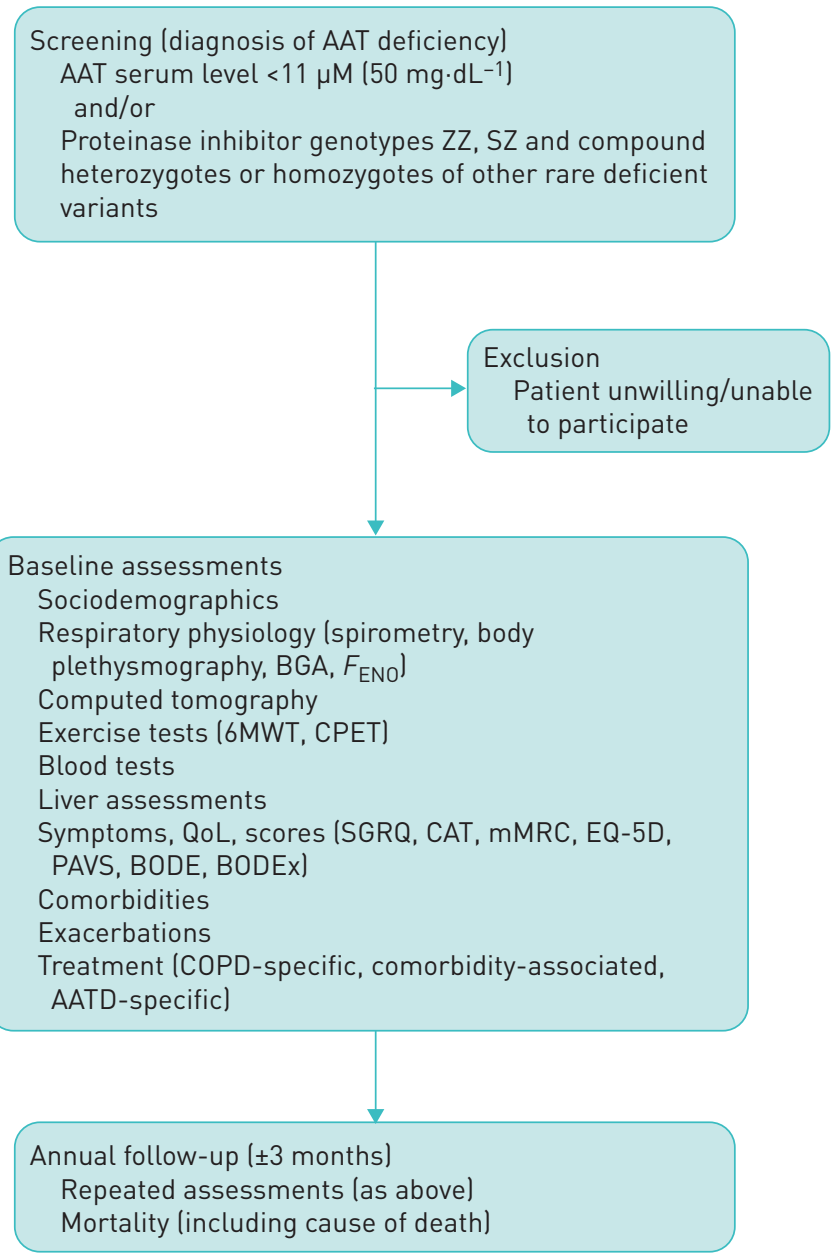

FIGURE 2 Baseline and follow-up assessments. Only demographics and information about lung and liver disease are mandatory. AAT: alpha-1 antitrypsin; BGA: blood gas analysis; $F_{\text {ENO }}$ : exhaled nitric oxide fraction; MWT: 6-min walk test; CPET: cardiopulmonary exercise testing: QoL: quality of life; SGRQ: St George's Respiratory Question-naire; CAT: COPD Assessment Test; mMRC: modified Medical Research Council dyspnoea scale; EQ-5D: EuroQoL 5-dimension quality of life questionnaire; PAVS: physical activity vital signs questionnaire; BODE: body mass index, obstruction, dyspnoea and exercise; BODEx: body mass index, obstruction, dyspnoea and exacerbations.

history (i.e. index case versus non-index case), AAT level, phenotype/genotype (b), information about AATD-related diseases (lung disease, liver disease, vasculitis and other AATD-related diseases).

\section{Respiratory physiology}

Spirometry equipment and performance of spirometric testing must conform to American Thoracic Society (ATS)/ERS standards [26]. Pre- and/or post-bronchodilator spirometry values will be collected at baseline, but post-bronchodilator values alone will be recorded at follow-up visits. Absolute values will be recorded, to enable the use of different reference equations to calculate the severity of impairment. In order to reduce the variability of observations, the same equipment must be used and the same staff member should evaluate and coach a given patient at each visit throughout the study, where possible.

For body plethysmography and diffusing capacity of the lung for carbon monoxide, international recommendations will be followed [27]. Arterial blood gas analysis will be measured using a quality controlled analyser. Measurements from the arterialised ear lobe are also acceptable [28]. The procedure for measurement of the fraction of exhaled nitric oxide will follow the ERS recommendations [29]

\section{Exercise tests}

The 6-min walk test will be performed according to the ATS guideline [30]. The patient's assessment of dyspnoea and fatigue will be captured using the Borg CR10 scale both before and after the test. Cardiopulmonary exercise testing will be performed according to the current recommendations [31]. 
Blood tests

Levels of liver enzymes, haematology values including red blood cells, platelets, leukocytes and white blood cells distribution, fibrinogen, C-reactive protein and hepatic virus titres will be measured and recorded in the database.

\section{Additional liver assessments}

One of two available ultrasound-based methods, either transient elastography or acoustic radiation force impulse [32] is recommended to assess early fibrosis of the liver. According to the methodology, the result will be recorded in $\mathrm{kPa}$ or $\mathrm{m} \cdot \mathrm{s}^{-1}$.

\section{Patient-reported outcomes: symptoms, quality of life, scores}

The commonest relevant respiratory symptoms of dyspnoea, cough and sputum production will be assessed. When the results of sputum culture are available, the bacterial strains isolated will be recorded. The CRF will also include fields dedicated to elevated liver enzymes and jaundice.

Several standardised questionnaires related to quality of life, symptoms and physical activity, available in multiple languages, will be used to collect information on patient-reported outcomes. These questionnaires will include the COPD assessment test [33], the modified Medical Research Council (mMRC) dyspnoea scale [34], the EuroQoL 5-dimension (EQ-5D) generic quality-of-life questionnaire [35] and the physical activity vital sign (PAVS) [36]. In some countries, the St George's Respiratory Questionnaire will be collected [37]. Patients will complete the questionnaires when rested and in a quiet area, and will be allowed to ask questions to obtain the truest or best response. The questionnaires will be collected and checked for completeness before the patient leaves the centre. Instrument scoring and handling of missing item data will be conducted in accordance with the user guidelines.

The body mass index, obstruction, dyspnoea and exercise (BODE) and the Body mass index, Obstruction, Dyspnoea and Exacerbations (BODEx) indices will be calculated automatically from entered and quality-controlled data [38, 39].

\section{Comorbidities}

Information on comorbidities (ischaemic heart disease, heart failure, pulmonary hypertension, peripheral artery disease, diabetes, arterial hypertension, osteoporosis, lung cancer and others) will be collected according to available information from the medical records. In some countries, an additional set of comorbidities will be recorded.

\section{Exacerbations of COPD}

An exacerbation is defined as an acute worsening of pre-existing respiratory symptoms that results in additional therapy [40]. COPD exacerbations are classified as mild or treated with increased use of short-acting bronchodilators only; moderate or treated with antibiotics and/or systemic corticosteroids; and severe requiring hospitalisation or visits the emergency room.

The following items will be collected: ambulatory and hospital-based exacerbations during the previous 12 months (total number, requiring systemic steroids, requiring antibiotics requiring new-onset non-invasive ventilation or intensive care unit admission, and main respiratory symptoms).

\section{Information on treatment}

The information collected will include the following items: bronchodilators, inhaled corticosteroids, oral corticosteroids, theophylline, phosphodiesterase-4 inhibitors, long-term oxygen therapy, long-term antibiotic treatment, noninvasive ventilation, pulmonary rehabilitation, vaccination, lung volume reduction and transplantation (lung, liver).

\section{Augmentation therapy}

The status of augmentation therapy (start, stop, dose, frequency, name of medication) will be assessed at baseline and at each follow-up visit.

\section{Discontinuation of follow-up}

If a patient is lost to follow-up, the reason for this will be assessed whenever possible. Patients who undergo lung or liver transplantation will be followed-up further. If a patient dies during follow-up, the cause of death, according to the treating physician, will be recorded. 


\section{Data review and database management}

Data collection

It is the investigator's responsibility to ensure adequate source documentation for all collected data. Designated investigator staff will enter the data required by the protocol into the CRFs. Spirometry reports (including the flow-volume curve) and CT reports may be uploaded after appropriate anonymisation. The investigator must certify that the data are complete and accurate by entering a password. After review for completeness, plausibility and correctness (flow-volume curve, assessed in uploaded curves) by the EARCO database monitor, the data will be locked.

\section{Database management and quality control}

It is the EARCO database manager's responsibility to review the CRF data entered by investigational staff for completeness and accuracy and instruct the site personnel to make any required corrections or additions. Queries will be sent to the investigational site. Designated investigator site staff are required to respond to the query and make any necessary changes to the data.

\section{Discussion}

In the last official statement about AATD from the ERS, the development of a European, follow-up registry (a longitudinal cohort) was identified by healthcare providers and patients as a research priority [12]. Registries have the potential to provide important insights into the natural history of rare diseases where epidemiological data cannot be derived from small-scale prospective cohort studies [41]. In developing the EARCO registry, we have followed guidance proposed in the EU and the extensive experience from other registries in the field of respiratory disease [24, 25, 41, 42]. According to these recommendations, it is essential to define a minimum required dataset that allows the collection of relevant information and limits the burden on investigators entering the data. Furthermore, the definitions across participating sites have to be harmonised to ensure that data are consistent and reliable. We will provide a shared data collection platform, and standardised definitions will be made available on the website (www.earco.eu) and upon request to all study sites.

Many different national registries on AATD exist throughout Europe [14, 17-23], but inclusion criteria, variables collected and level of quality control are different. The EARCO registry will harmonise the collection of prospective data, and its design will allow the new prospectively recorded data to be linked with the historical data collected in the different national registries. In order to do that, the EARCO registry will include the sum of all variables included in all national European registries, but some of them will only be mandatory for patients in a given country where these variables were collected historically. With this design, EARCO will consist of a set of common mandatory fields and a series of variables that will be mandatory at a national level.

The inclusion and exclusion criteria are deliberately broad to allow the inclusion of a large and varied population representative of "real-life" AATD patients and management across Europe.

As an example of variables to be collected, lung function tests are used in the assessment of the severity, progression and prognosis of COPD [40]. Most studies of lung function decline in COPD are based on $\mathrm{FEV}_{1}$, because it shows the least variability. However, the main pathological marker of the progression of the lung disease of AATD is an increase in the destruction of the lung parenchyma, as shown most specifically by CT densitometry $[8,43]$. However, lung densitometry requires careful standardisation and is not yet ready for use in routine clinical practice [44-46]. EARCO may therefore represent a platform for the development of future multicentre studies using CT densitometry to evaluate the evolution of emphysema in large cohorts of patients with AATD in usual clinical practice [47]. In contrast, other lung function derived markers that have the potential to reflect the magnitude and evolution of emphysema are gas transfer and the hallmarks of hyperinflation [48]. Therefore, diffusing capacity of the lung for carbon monoxide and body plethysmographic measurements are measured routinely in the majority of participating countries, according to the most recent international recommendations [49-51].

We consider a biobank as an important objective for EARCO. However, in the first step we have to overcome the legal and organisational hurdles that are associated with an international database. As these hurdles are even higher for an international biobank we will apply for additional funding in a second step and try to organise/build a biobank in the future.

The participation of patients, coordinated by the European Lung Foundation and Alpha-1 Global has been crucial for the development of a CRF that takes into consideration not only physiological variables, but also the variables that have recognised impact and importance patients. Therefore, the inclusion of patient-reported outcomes was essential. However, although different patient-reported outcomes have been used in patients with AATD [52], there has been little formal validation of them in this population. We 
therefore included patient-reported outcomes validated in COPD [53]. The EQ-5D is a simple but effective standardised instrument designed for use as a measure of health outcome. It provides both a compact descriptive profile and a single index value that can be used in the clinical and economic evaluation of health care [35]. The COPD assessment test has been developed as a simpler tool to assess quality of life and symptom burden in COPD patients specifically [33]. The mMRC is an easy-to-use instrument designed for the quantification of dyspnoea [54]. The latter two questionnaires are also part of the multidimensional categories of COPD severity [40]. PAVS is a brief $(<30 \mathrm{~s})$ physical activity questionnaire intended to be administered to patients just as vital signs, and height and weight are measured [55]. PAVS is intended to assess how much light, moderate or vigorous physical activity a person performs in a typical week. The assessment facilitates patient counselling about physical activity [56]. Composite scores that combine different physiological measures are used to assess the severity and prognosis of obstructive lung disease and liver disease, but the experience in AATD is very limited [57]. We will use the BODE [38] and BODEx [39] for lung disease and the Fibrosis-4 [57] for liver disease to assess their values in monitoring and determining long-term prognosis in AATD.

The EARCO registry is not the first European registry in AATD. The Alpha One International Registry (AIR) group included representatives from 14 European and some non-European countries [58]. AIR was very successful in collecting baseline data. However, due to limited funding, longitudinal data could not be collected systematically, and the quality of existing data (completeness and accuracy) may be regarded as suboptimal. This can be partially explained by the fact that data were not processed centrally for data clearing and data control [59]. The EARCO registry will collaborate closely with existing national AATD registries and will harmonise the process of data collection and assess the quality of the data included prospectively, thus overcoming the limitations present in AIR.

The target sample size is based on experiences with similar projects. A short-term target of 1000 patients in the first year of the project after database setup has been set. To ascertain representativeness for the European population we aim to include $>3000$ patients from $\geqslant 20$ European countries by the end of the third year.

Acknowledging the imminent limitations of a non-interventional cohort study, the EARCO registry itself will not provide an answer to all the research questions in the field of AATD, but it can be the backbone of different research initiatives, some of which have already been highlighted [15].

Acknowledgements: We want to acknowledge the support from Elise Heuvelin, Céline Genton and Alessandra Marguerat (European Respiratory Society, Lausanne, Switzerland) in the logistics and organisation of EARCO; Barbara Johnson (European Lung Foundation, Sheffield, UK) for her support connecting to different patient organisations; Eduardo Loeb (Pneumology Dept, University Hospital Vall d'Hebron/VHIR, Barcelona, Spain) for his contribution as data manager of EARCO; and Joanna Shakespeare (Dept of Respiratory and Sleep Sciences, University Hospitals Coventry and Warwickshire NHS Trust, Walsgrave, UK) for her help in designing the database.

Support statement: The EARCO registry is supported by the European Respiratory Society, Kamada, pH Pharma, CSL Behring and Grifols. Funding information for this article has been deposited with the Crossref Funder Registry.

Conflict of interest: T. Greulich reports grants from CSL-Behring, Grifols and Kamda during the conduct of the study; personal fees for lectures and advisory boards from AstraZeneca, Berlin-Chemie, Boehringer Ingelheim, Chiesi, CSL-Behring, GSK and Novartis, grants and personal fees for lectures and advisory boards from Grifols, and grants from the German Centre for Lung Research (DZL), outside the submitted work. A. Altraja reports travel support to attend the EARCO stakeholder board meeting on 17 June 2019 from EARCO during the conduct of the study; support for clinical trials and honoraria for advisory board meetings from CSL Behring, honoraria for lectures/chairmanships and advisory board meetings and travel support from Shire Pharmaceuticals, AstraZeneca, Boehringer Ingelheim, Chiesi (Norameda), GlaxoSmithKline, MSD, Novartis, Roche, Bayer and Actelion (Johnson \& Johnson), research support from Pfizer, travel support from Teva, Sanofi and United Therapeutics (AOP Orphan), honoraria for lectures and travel support from Berlin-Chemie Menarini Group, and honoraria for lectures from Orion, outside the submitted work, M. Barrecheguren reports speaker fees from Menarini, Grifols, CSL Behring, Boehringer Ingelheim, Gebro Pharma and GlaxoSmithKline, and consulting fees from Novartis and GlaxoSmithKline, outside the submitted work. R. Bals reports grants and personal fees from AstraZeneca and Boehringer Ingelheim, personal fees from GlaxoSmithKline and Grifols, grants and personal fees from Novartis, personal fees from CSL Behring, grants from the German Federal Ministry of Education and Research (BMBF) Competence Network Asthma and COPD (ASCONET), Sander Stiftung, Schwiete Stiftung, Krebshilfe and Mukoviszidose e.V., outside the submitted work. J. Chlumsky reports personal fees for lectures and consultation from CSL Behring, and travel support from CSL Behring, outside the submitted work. J. Chorostowska-Wynimko reports grants, personal fees and nonfinancial support from Grifols, personal fees from Kamada, grants, personal fees and nonfinancial support from AstraZeneca, personal fees and nonfinancial support from Pfizer, MSD and BMS, personal fees from GSK, Novartis amd Chiesi, personal fees and nonfinancial support from Roche, grants and personal fees from Boehringer Ingelheim, personal fees and nonfinancial support from Abbvie, grants, personal fees and nonfinancial support from CSL Behring, personal fees from Lekam, grants, personal fees and nonfinancial support from CelonPharma, and personal fees from Takeda, outside the submitted work. C. Clarenbach reports consulting fees and travel support from Vifor Pharma, and consulting or speaker fees from Boehringer, Novartis, GSK, AstraZeneca and Sanofi, outside the submitted work. L. Corda has nothing to disclose. A.G. Corsico has nothing to disclose. I. Ferrarotti has nothing to disclose. C. Esquinas has nothing to disclose. C. Gouder has nothing to disclose. 
A. Hećimović reports speaker fees from Roche, Boehringer Ingelheim, Novartis and MSD, outside the submitted work. A. Ilic has nothing to disclose. Y. Ivanov has nothing to disclose. S. Janciauskiene has nothing to disclose. W. Janssens reports receiving research grants and money for consultancy activities from Behring, AstraZeneca, Boerhinger Ingelheim, GSK, Chiesi, outside the submitted work. He is co-founder of ArtiQ, a spinoff company in healthcare. M. Kohler reports advisor fees from Novartis, Boehringer Ingelheim, AstraZeneca, GSK and Bayer, outside the submitted work. A. Krams reports personal fees and nonfinancial support from AstraZeneca, Berlin-Chemie/Menarini and Boehringer Ingelheim, personal fees from GlaxoSmithKline and Merck Serono, personal fees and nonfinancial support from Norameda (represents Chiesi in Baltic countries), personal fees from Novartis, and nonfinancial support from Mylan, outside the submitted work. B. Lara has nothing to disclose. R. Mahadeva reports personal fees from Chiesi, Astra Zeneca and Boehringer Ingelheim, and grants from Pfizer Open Air, outside the submitted work. G. McElvaney has sat on advisory boards for CSL Behring, Grifols, Vertex and Chiesi. J-F. Mornex reports nonfinancial support from ADAAT, grants, personal fees and nonfinancial support from LFB and CSL Behring, nonfinancial support from Grifols, personal fees and nonfinancial support from Polyphor, during the conduct of the study; personal fees and nonfinancial support from Actelion, GSK, Roche and Novartis, nonfinancial support from Bayer, personal fees and nonfinancial support from BMS, nonfinancial support from Pfizer and MSD, personal fees from Ellivie, and nonfinancial support from Boehringer, outside the submitted work. K. O'Hara reports nonfinancial support from ELF/ ERS and Mereo BioPharma Group PLC, grants from CSL Behring, and also receives donations from individuals/ companies as a result of fundraising activities; personal fees and nonfinancial support from NICE and nonfinancial support from Alpha-1 Global, outside the submitted work. D. Parr reports advisory board and consultancy fees from CSL Behring and Kamada, outside the submitted work. E. Piitulainen has nothing to disclose. K. Schmid-Scherzer has nothing to disclose. N. Seersholm has nothing to disclose. R.A. Stockley reports a grant for a small, investigator-lead project and personal fees for a trial steering board from CSL Behring; a lecture fee from GSK; advisory board fees from Vertex, inhbrix, Mereobiopharma and Novartis; and lecture fees from Grifols, during the conduct of the study. He acts in an advisory manner for several companies with an interest in $\alpha_{1}$-antitrypsin deficiency. J. Stolk has nothing to disclose. M. Sucena reports lecture fees and travel pay from Grifols and CSL Behring, outside the submitted work. H. Tanash has nothing to disclose. A. Turner reports grants and personal fees from CSL Behring; personal fees and nonfinancial support from Boehringer Ingelheim; nonfinancial support from GSK; grants, personal fees and nonfinancial support from AstraZeneca; grants, personal fees and nonfinancial support from Chiesi; grants from Grifols biotherapeutics, outside the submitted work. R. Ulmeanu has nothing to disclose. M. Wilkens reports that as chairman of the patient organisation Alpha1 Deutschland e.V., he does not receive personally any donations but his organisation receives money from public funds as well as from the pharmaceutical industry, including grants and travelling costs. A. Yorgancioğlu reports grants from MSD, and personal fees from GSK, AstraZeneca, Abdi Ibrahim, Chiesi, Novartis and Sandoz, outside the submitted work. A. Zaharie has nothing to disclose. M. Miravitlles reports speaker fees from AstraZeneca, Boehringer Ingelheim, Chiesi, Cipla, AstraZeneca, Menarini, Rovi, Bial, Zambon, CSL Behring, Grifols and Novartis, consulting fees from AstraZeneca, Boehringer Ingelheim, Chiesi, GlaxoSmithKline, Bial, Gebro Pharma, CSL Behring, Laboratorios Esteve, Ferrer, Mereo Biopharma, Verona Pharma, TEVA, pH Pharma, Novartis and Grifols, and research grants to his institution from GlaxoSmithKline and Grifols, outside the submitted work.

\section{References}

1 Silverman EK, Sandhaus RA. Clinical practice. Alpha1-antitrypsin deficiency. N Engl J Med 2009; 360: 2749-2757.

2 Blanco I, Bueno P, Diego I, et al. Alpha-1 antitrypsin $\mathrm{Pi}^{\star} \mathrm{Z}$ gene frequency and $\mathrm{Pi}^{\star} \mathrm{ZZ}$ genotype numbers worldwide: an update. Int J Chron Obstruct Pulmon Dis 2017; 12: 561-569.

3 Eriksson S. Alpha 1-antitrypsin deficiency: lessons learned from the bedside to the gene and back again. Historic perspectives. Chest 1989; 95: 181-189.

4 Lomas DA, Evans DL, Finch JT, et al. The mechanism of $\mathrm{Z}$ alpha 1-antitrypsin accumulation in the liver. Nature 1992; 357: 605-607.

5 Stockley RA, Miravitlles M, Vogelmeier C. Augmentation therapy for alpha-1 antitrypsin deficiency: towards a personalised approach. Orphanet J Rare Dis 2013; 8: 149.

6 Molloy K, Hersh CP, Morris VB, et al. Clarification of the risk of chronic obstructive pulmonary disease in $\alpha 1$-antitrypsin deficiency PiMZ heterozygotes. Am J Respir Crit Care Med 2014; 189: 419-427.

7 Foreman MG, Wilson C, DeMeo DL, et al. Alpha-1 antitrypsin PiMZ genotype is associated with chronic obstructive pulmonary disease in two racial groups. Ann Am Thorac Soc 2017; 14: 1280-1287.

8 Dirksen A, Piitulainen E, Parr DG, et al. Exploring the role of CT densitometry: a randomised study of augmentation therapy in alpha1-antitrypsin deficiency. Eur Respir J 2009; 33: 1345-1353.

9 Stockley RA, Parr DG, Piitulainen E, et al. Therapeutic efficacy of $\alpha-1$ antitrypsin augmentation therapy on the loss of lung tissue: an integrated analysis of 2 randomised clinical trials using computed tomography densitometry. Respir Res 2010; 11: 136.

10 Chapman KR, Burdon JG, Piitulainen E, et al. Intravenous augmentation treatment and lung density in severe $\alpha 1$ antitrypsin deficiency (RAPID): a randomised, double-blind, placebo-controlled trial. Lancet 2015; 386: 360-368.

11 Gøtzsche PC, Johansen HK. Intravenous alpha-1 antitrypsin augmentation therapy for treating patients with alpha-1 antitrypsin deficiency and lung disease. Cochrane Database Syst Rev 2010; 7: CD007851.

12 Miravitlles M, Dirksen A, Ferrarotti I, et al. European Respiratory Society statement: diagnosis and treatment of pulmonary disease in $\alpha_{1}$-antitrypsin deficiency. Eur Respir J 2017; 50: 1700610.

13 Horváth I, Canotilho M, Chlumský J, et al. Diagnosis and management of alpha1-antitrypsin deficiency in Europe: an expert survey. ERJ Open Res 2019; 5: 00171-02018.

14 Ferrarotti I, Baccheschi J, Zorzetto $\mathrm{M}$, et al. Prevalence and phenotype of subjects carrying rare variants in the Italian registry for alpha1-antitrypsin deficiency. J Med Genet 2005; 42: 282-287.

15 Miravitlles M, Chorostowska-Wynimko J, Ferrarotti I, et al. The European Alpha-1 Research Collaboration (EARCO): a new ERS Clinical Research Collaboration to promote research in alpha-1 antitrypsin deficiency. Eur Respir J 2019; 53: 1900138.

16 Brightling C, Genton C, Bill W, et al. ERS Clinical Research Collaborations: underpinning research excellence. Eur Respir J 2018; 52: 1801534.

17 Stockley RA. Antitrypsin Deficiency Assessment and Programme for Treatment (ADAPT): the United Kingdom registry. COPD 2015; 12: Suppl. 1, 63-68. 
18 Bals R, Vogelmeier C. Alpha 1-Antitrypsin-Labor und Alpha 1-Antitrypsin-Register [Alpha-1-antitrypsin laboratory and German alpha-1-antitrypsin registry]. Pneumologie 2004; 58: 637-638.

19 Lara B, Blanco I, Martínez MT, et al. Spanish registry of patients with alpha-1 antitrypsin deficiency: database evaluation and population analysis. Arch Bronconeumol 2017; 53: 13-18.

20 Chorostowska-Wynimko J, Struniawski R, Sliwinski P, et al. The national alpha-1 antitrypsin deficiency registry in Poland. COPD 2015; 12: Suppl. 1, 22-26.

21 Carroll TP, O'Connor CA, Floyd O, et al. The prevalence of alpha-1 antitrypsin deficiency in Ireland. Respir Res 2011; 12: 91.

22 Seersholm N, Kok-Jensen A, Dirksen A. Survival of patients with severe alpha 1-antitrypsin deficiency with special reference to non-index cases. Thorax 1994; 49: 695-698.

23 Piitulainen E, Tanash HA. The clinical profile of subjects included in the Swedish national register on individuals with severe alpha 1-antitrypsin deficiency. COPD 2015; 12: Suppl. 1, 36-41.

24 European Medicines Agency. Discussion paper: use of patient disease registries for regulatory purposes methodological and operational considerations. www.ema.europa.eu/en/documents/other/discussion-paper-use-patientdisease-registries-regulatory-purposes-methodological-operational_en.docx Date last updated: November 5, 2018.

25 Bilton D, Caine N, Cunningham S, et al. Use of a rare disease patient registry in long-term post-authorisation drug studies: a model for collaboration with industry. Lancet Respir Med 2018; 6: 495-496.

26 Miller MR, Hankinson J, Brusasco V, et al. Standardisation of spirometry. Eur Respir J 2005; 26: 319-338.

27 Criée CP, Sorichter S, Smith HJ, et al. Body plethysmography - its principles and clinical use. Respir Med 2011; 105: 959-971.

28 Pitkin AD, Roberts CM, Wedzicha JA. Arterialised earlobe blood gas analysis: an underused technique. Thorax 1994; 49: 364-366.

29 American Thoracic Society, European Respiratory Society. ATS/ERS recommendations for standardized procedures for the online and offline measurement of exhaled lower respiratory nitric oxide and nasal nitric oxide, 2005. Am J Respir Crit Care Med 2005; 171: 912-930.

30 ATS Committee on Proficiency Standards for Clinical Pulmonary Function Laboratories. ATS statement: guidelines for the six-minute walk test. Am J Respir Crit Care Med 2002; 166: 111-117.

31 Guazzi M, Arena R, Halle M, et al. 2016 focused update: clinical recommendations for cardiopulmonary exercise testing data assessment in specific patient populations. Circulation 2016; 133: e694-e711.

32 Ferraioli G, Filice C, Castera L, et al. WFUMB guidelines and recommendations for clinical use of ultrasound elastography: Part 3: liver. Ultrasound Med Biol 2015; 41: 1161-1179.

33 Jones PW, Harding G, Berry P, et al. Development and first validation of the COPD Assessment Test. Eur Respir J 2009; 34: 648-654

34 Bestall JC, Paul EA, Garrod R, et al. Usefulness of the Medical Research Council (MRC) dyspnoea scale as a measure of disability in patients with chronic obstructive pulmonary disease. Thorax 1999; 54: 581-586.

35 EuroQol Group. EuroQol - a new facility for the measurement of health-related quality of life. Health Policy 1990 16: $199-208$.

36 Greenwood JL, Joy EA, Stanford JB. The Physical Activity Vital Sign: a primary care tool to guide counseling for obesity. J Phys Act Health 2010; 7: 571-576.

37 Jones PW, Quirk FH, Baveystock CM. The St George's Respiratory Questionnaire. Respir Med 1991; 85: Suppl. B, $25-31$.

38 Celli BR, Cote CG, Marin JM, et al. The body-mass index, airflow obstruction, dyspnea, and exercise capacity index in chronic obstructive pulmonary disease. N Engl J Med 2004; 350: 1005-1012.

39 Espantoso-Romero M, Román Rodríguez M, Duarte-Pérez A, et al. External validation of multidimensiona prognostic indices (ADO, BODEx and DOSE) in a primary care international cohort (PROEPOC/COPD cohort) BMC Pulm Med 2016; 16: 143.

40 Vogelmeier CF, Criner GJ, Martinez FJ, et al. Global Strategy for the Diagnosis, Management, and Prevention of Chronic Obstructive Lung Disease 2017 Report: GOLD Executive Summary. Eur Respir J 2017; 49: 1900164.

41 Viviani L, Zolin A, Mehta A, et al. The European Cystic Fibrosis Society Patient Registry: valuable lessons learned on how to sustain a disease registry. Orphanet J Rare Dis 2014; 9: 81.

42 Chalmers JD, Aliberti S, Polverino E, et al. The EMBARC European Bronchiectasis Registry: protocol for an international observational study. ERJ Open Res 2016; 2: 00081-02015.

43 Dowson LJ, Guest PJ, Stockley RA. Longitudinal changes in physiological, radiological, and health status measurements in $\alpha_{1}$-antitrypsin deficiency and factors associated with decline. Am J Respir Crit Care Med 2001; 164: 1805-1809.

44 Parr DG, Stockley RA. Standardization of CT densitometry. Radiology 2004; 230: 887.

45 Parr DG, Stoel BC, Stolk J, et al. Influence of calibration on densitometric studies of emphysema progression using computed tomography. Am J Respir Crit Care Med 2004; 170: 883-890.

46 Subramanian DR, Gupta S, Burggraf D, et al. Emphysema- and airway-dominant COPD phenotypes defined by standardised quantitative computed tomography. Eur Respir J 2016; 48: 92-103.

47 Crossley D, Renton M, Khan M, et al. CT densitometry in emphysema: a systematic review of its clinical utility. Int J Chron Obstruct Pulmon Dis 2018; 13: 547-563.

48 Mohamed Hoesein FA, Zanen P, van Ginneken B, et al. Association of the transfer coefficient of the lung for carbon monoxide with emphysema progression in male smokers. Eur Respir J 2011; 38: 1012-1018.

49 Macintyre N, Crapo RO, Viegi G, et al. Standardisation of the single-breath determination of carbon monoxide uptake in the lung. Eur Respir J 2005; 26: 720-735.

50 Miller MR, Crapo R, Hankinson J, et al. General considerations for lung function testing. Eur Respir J 2005; 26 $153-161$.

51 Wanger J, Clausen JL, Coates A, et al. Standardisation of the measurement of lung volumes. Eur Respir J 2005; 26 511-522.

52 Manca S, Rodriguez E, Huerta A, et al. Usefulness of the CAT, LCOPD, EQ-5D and COPDSS scales in understanding the impact of lung disease in patients with alpha-1 antitrypsin deficiency. COPD 2014; 11: 480-488.

53 Jones $\mathrm{P}$, Miravitlles M, van der Molen T, et al. Beyond FEV 1 in COPD: a review of patient-reported outcomes and their measurement. Int J Chron Obstruct Pulmon Dis 2012; 7: 697-709. 
54 Mahler DA, Wells CK. Evaluation of clinical methods for rating dyspnea. Chest 1988; 93: 580-586.

55 Ball TJ, Joy EA, Gren LH, et al. Concurrent validity of a self-reported physical activity "vital sign" questionnaire with adult primary care patients. Prev Chronic Dis 2016; 13: E16.

56 Strath SJ, Kaminsky LA, Ainsworth BE, et al. Guide to the assessment of physical activity: clinical and research applications: a scientific statement from the American Heart Association. Circulation 2013; 128: 2259-2279.

57 Vallet-Pichard A, Mallet V, Nalpas B, et al. FIB-4: an inexpensive and accurate marker of fibrosis in HCV infection. Comparison with liver biopsy and fibrotest. Hepatology 2007; 46: 32-36.

58 Luisetti M, Miravitlles M, Stockley RA. Alpha1-antitrypsin deficiency: a report from the 2nd meeting of the Alpha One International Registry, Rapallo (Genoa, Italy), 2001. Eur Respir J 2002; 20: 1050-1056.

59 Stockley RA, Luisetti M, Miravitlles M, et al. Ongoing research in Europe: Alpha One International Registry (AIR) objectives and development. Eur Respir J 2007; 29: 582-586. 